\title{
Lack of adherence to hypertension treatment guidelines among GPs in southern Sweden-A case report-based survey
}

\author{
Rickard Ekesbo ${ }^{1,2,4^{*}}$, Patrik Midlöv ${ }^{1,2}$, Sofia Gerward ${ }^{3}$, Kristin Persson ${ }^{1}$, Christina Nerbrand ${ }^{2}$ and Lennart Johansson ${ }^{2}$
}

\begin{abstract}
Background: General practitioners (GPs) often fail to correctly adhere to guidelines for the treatment of hypertension. The reasons for this are unclear, but could be related to lack of knowledge in assessing individual patients' cardiovascular disease risk. Our aim was to investigate how GPs in southern Sweden adhere to clinical guidelines for the treatment of hypertension when major cardiovascular risk factors are taken into consideration.
\end{abstract}

Method: A questionnaire with five genuine cases of hypertension with different cardiovascular risk profiles was sent to a random sample of GPs in southern Sweden $(n=109)$ in order to investigate the attitude towards blood pressure (BP) treatment when major cardiovascular risk factors were present.

Results: In general, GPs who responded tended to focus on the absolute target BP rather than assessing the entire cardiovascular risk factor profile. Thus, cases with the highest risk of cardiovascular disease were not treated accordingly. However, there was also a tendency to overtreat the lowest risk individuals. Furthermore, the BP levels for initiating pharmacological treatment varied widely (systolic BP $140-210 \mathrm{mmHg}$ ). ACE inhibitors (70\%) were the most common first choice of pharmacological treatment.

Conclusion: In this study, GPs in Southern Sweden were suggesting, for different cases, either under- or overtreatment in relation to current guidelines for treatment of hypertension. On reason may be that they failed to correctly assess individual cardiovascular risk factor profiles.

Key points: Despite international and national clinical guidelines on the treatment of hypertension, general practitioners often fail to correctly assess the cardiovascular risk for patients in a clinical setting.

- Most GPs use target blood pressure levels but do not consider other cardiovascular risk factors.

- Both under- and overtreatment of high and low cardiovascular risk groups were seen in this study.

Keywords: Hypertension, Adherence, Guidelines, Treatment, Primary care

\section{Background}

High blood pressure (BP) is an important risk factor for cardiovascular disease [1]. In accordance with official guidelines [2], the overall cardiovascular risk should be taken into account before pharmacological treatment of high BP begins. There are a number of risk factors for developing manifest cardiovascular

\footnotetext{
* Correspondence: rickard.ekesbo@skane.se

${ }^{1}$ General Practice/Family Medicine, Department of Clinical Sciences, Faculty of Medicine, Lund University, Malmö, Sweden

${ }^{2}$ The R\&D Department of Primary Care, Malmö, Region Skåne, Sweden

Full list of author information is available at the end of the article
}

disease, most important of which are age, family history, gender, smoking, diabetes, hypercholesterolemia, low physical activity and low consumption of fruit and vegetables [3].

In spite of clear guidelines on the treatment of high $\mathrm{BP}$ and the availability of a number of tools for risk assessment, there is large variation in the way that different GPs initiate treatment for high BP. Furthermore, a large proportion of patients with high $\mathrm{BP}$ receive no or inadequate treatment [4].

In a previous study from our group, only $20 \%$ of patients treated from hypertension in primary care in southern

\section{C) Biomed Central}

(c) 2012 Ekesbo et al.; licensee BioMed Central Ltd. This is an Open Access article distributed under the terms of the Creative Commons Attribution License (http://creativecommons.org/licenses/by/2.0), which permits unrestricted use, distribution, and reproduction in any medium, provided the original work is properly cited. 
Sweden reached the currently recommended target levels $(<140 / 90 \mathrm{mmHg})$ [5]. This result illustrated the need for continued follow-up of defined groups of patients in order to improve the quality of care.

Furthermore, three main barriers towards implementing guidelines were identified among GPs: doubt concerning patient motivation, patient age and absence of other risk factors [6].

These previous findings reflect the problem that while guidelines are clear and concise, real patients are, in their nature, much more complex. The aim of this study was to investigate how GPs in southern Sweden adhere to clinical guidelines for the treatment of hypertension, using a case-based questionnaire to reflect patients' complex nature and taking major cardiovascular risk factors into account.

\section{Methods}

Participants

Skåne County is situated in the southernmost part of Sweden and has approximately 1,150,000 inhabitants. Primary care is provided by approximately 600 GPs, of whom 420 are employed by public primary health care centres. Of these 90 primary care centres, 24 centres (employing a total of 109 GPs) were randomly selected for participation in the survey and is thus believed to be a cross-section of the GP's of the area. An invitation letter, including self-administered questionnaires (see below) was sent to the head of each primary health care centre during 2006. Non-responders received telephone reminders. For each primary health care centre agreeing to participate in the survey, all physicians working at the centre were asked to fill out questionnaires on attitudes towards current guidelines $[1,2]$ and general practice in the treatment of high BP.

No data were collected for the 19 GPs who did not fill in the questionnaires.

\section{Questionnaires}

The survey included two postal questionnaires. Sex and information on professional experience was recorded for all GPs. The results of the first questionnaire have previously been published [6]. The second questionnaire included four questions based on five genuine cases of high BP with different cardiovascular risk profiles (see below). The five cases were based on true cases and intended to represent reasonable variation of hypertensive patients with different risk profiles common in primary care practice. Thus, the cases represent various levels of risk rather than being absolutely representative for the hypertensive population of the region. Such a cohort could possibly present a different pattern but might also give a less clear picture for decision-making regarding hypertension.
The following four specific questions were used for each case:

1. Do you find that drug treatment is indicated for this patient?

2. If so, what is your first choice of drug class for treatment?

3. What is your target BP?

4. What other cardiovascular disease (CVD) risk factors do your think should be considered when choosing an intervention?

Possible answers to the first question were "yes" or "no". The second question included six alternative answers: "beta blockers", "diuretics", "ACE inhibitors", "angiotensin receptor blockers (ARBs)", "calcium channel blockers" and "other". Questions 3 and 4 were open, without predefined answers to choose from; however reported CVD risk factors (question 4) were later categorized into four groups: hyperglycemia or diabetes, smoking, hyperlipidemia and obesity.

Data for each case were registered separately. The cases were assessed using two different risk assessment instruments: the SCORE, which is intended to assess cardiovascular disease mortality risk in patients without manifest cardiovascular disease and which is based on subject gender, age, systolic blood pressure, smoking status and cholesterol levels [2]; and the joint European Society of Hypertension (ESH) and European Society of Cardiology (ESC) risk assessment instruments, which take into account other risk factors and which also cover patients with manifest cardiovascular complications [1].

\section{Statistical methods}

Only descriptive data were used in this study.

\section{Results}

The results are presented as individual cases, followed by interpretation of the results and adherence to guidelines according to the SCORE and ESC scoring systems. A short version is shown in Table 1 .

\section{Case 1 facts: female, age 45 years, BP 160/95}

Family history of cardiovascular disease. Non-smoker. Married with two children. Administrative work with low stress. Exercises three days a week. Previously had high blood pressure about 10 years ago, as measured by her occupational health physician and received drug treatment for a short period. BMI: $25 \mathrm{~kg} / \mathrm{m}^{2}$. ECG: normal. Routine tests (full blood count and routine biochemistry): no abnormalities detected (NAD). Serum cholesterol: $5.6 \mathrm{mmol} / \mathrm{l}$. 
Table 1 The cases in short form with risk factors and response rate for treatment

\begin{tabular}{|c|c|c|c|c|c|c|c|c|}
\hline Case & SBP/DBP & Diabetes & $\begin{array}{l}\text { Total } \\
\text { Cholesterol } \\
\end{array}$ & $\begin{array}{l}\text { Target organ } \\
\text { damage }\end{array}$ & Heredity & Score & ESC & $\begin{array}{l}\text { Proportion } \\
\text { treating (\%) }\end{array}$ \\
\hline 1 & $160 / 90$ & 0 & 5.6 & 0 & 0 & $0-1$ & 1 & 92 \\
\hline 2 & $160 / 95$ & 0 & 6.7 & $\mathrm{LVH}$ & 0 & 2 & 2 & 99 \\
\hline 3 & $150 / 80$ & + & 5.0 & 0 & 0 & NA & 2 & 94 \\
\hline 4 & $150 / 80$ & 0 & 4.0 & CABG & + & $N A$ & 3 & 70 \\
\hline 5 & $155 / 85$ & 0 & 6.2 & $?$ & $?$ & NA & 2 & 58 \\
\hline
\end{tabular}

$\mathrm{LVH}=$ Left ventricular hypertrophy. CABG = Coronary artery bypass graft.

\section{Case 1 risk assessment}

Case 1 represents a case of low CVD risk, being a nonsmoking female with mild hypertension. According to the risk assessment, case no 1 has a SCORE of $0-1$ and an ESC score of 1. Thus, the risk for manifest CVD disease within 10 years is very low (1-2\%) and blood pressure treatment may not be indicated.

\section{Case 1 results}

In this case, $92 \%$ of the GPs found a drug treatment indication for hypertension. The target systolic blood pressure was 140 for $83 \%$ of responders and the diastolic blood pressure 90 in $46 \%$ of responders.

Among those who found a drug treatment indication, $73 \%$ selected diuretics as their first choice of treatment and 25\% ACE inhibitors or ARBs. 28 and 29\% of GPs, respectively, thought that the cholesterol levels and the weight also should be treated.

\section{Case 2 facts: female, age 54 years, BP 160/95}

No family history of cardiovascular disease. Non-smoker. Married with two children. Originally from Greece. Manual worker. Slight obesity. No daily exercise. Blood pressure repeatedly measured to be $160 / 95$. Patient reports occasional palpitations and decreasing physical condition. Physical examination reveals a murmur in the left carotid artery. BMI: $30 \mathrm{~kg} / \mathrm{m}^{2}$. ECG: suspected left ventricular hypertrophy (LVH). Routine tests: NAD. Serum-cholesterol: $6.7 \mathrm{mmol} / \mathrm{l}$.

\section{Case 2 risk assessment}

This patient represents a medium cardiovascular risk, having suspected left ventricular hypertrophy. She has a SCORE of 2 and an ESC score of 2 . With hypertension in the medium range, this patient should be treated. Target organ damage (TOD) should be addressed.

\section{Case 2 results}

Almost all GPs (99\%) chose to treat the blood pressure. Of these, 43\% selected ACE-I as their first choice of treatment, followed by diuretics $(23 \%)$ and beta blockers (10\%). $41 \%$ of the GPs thought that the raised serum cholesterol should be treated.
Case 3 facts: male, age 56 years, BP 150/80

Family history of diabetes, but not heart disease. Nonsmoker. Married with two children. Taxi driver. Low exercise levels. Diabetes since the age of 5; treated with diet and oral antidiabetics; no organ complications. Blood pressure measured to be $150 / 80$ (by his GP) and $145 / 80$ (by his diabetes nurse). BMI: $28 \mathrm{~kg} / \mathrm{m}^{2}$. ECG: normal. Routine tests: NAD. Serum creatinine: $74 \mathrm{mmol} / \mathrm{l}$. Microalbuminuria: negative. Serum cholesterol: 5,0 mmol/l. HgbA1c: $6.8 \%$.

\section{Case 3 risk assessment}

This case represents a medium cardiovascular risk with diabetes and an unsatisfactory blood pressure. Due to the presence of diabetes mellitus type 2 SCORE cannot be assessed. The ESC score is 2 .

\section{Case 3 results}

94\% of the GPs wanted to treat the hypertension, the majority of whom (79\%) selected ACE inhibitors as the first choice of treatment. The target BP levels for most was $130 / 80$, which is the recommended value for diabetic patients [1]. 26 and $22 \%$ of the GPs, respectively, wanted to increase the treatment of hyperglycemia and hyperlipidemia, whereas $41 \%$ wished to treat the patient's overweight.

\section{Case 4 facts: female, age 62 years, BP 150/80}

Family history of coronary heart disease on the maternal and paternal sides. Married. Newspaper delivery person. Coronary artery bypass graft (CABG) five years ago due to angina problems. Current medication: metoprolol $50 \mathrm{mg} \times 1$, pravastatin $40 \mathrm{mg} \times 1$, felodipin $10 \mathrm{mg} \times 1$ and aspirin $75 \mathrm{mg} \times 1$. Smokes 20 cigarettes per day. No current angina. Home-measured BP around 140/80; nurse-measured blood pressure 150/80; GP-measured BP 145/80. BMI: 25 kg/m². ECG: normal. Routine tests: NAD. Serum creatinine: 67. Microalbuminuria: negative. Serum cholesterol: $4.0 \mathrm{mmol} / \mathrm{l}$.

\section{Case 4 risk assessment}

This is a case of high cardiovascular risk due to manifest coronary artery disease, continued smoking, and elevated blood pressure. SCORE cannot be assessed as manifest coronary disease is present. The ESC score is 3. 


\section{Case 4 results}

$70 \%$ of GPs wanted to increase hypertension treatment, with ACE inhibitors being the most common choice (55\%), followed by thiazides (28\%). Smoking was identified as a treatable risk factor by $85 \%$ of GPs.

\section{Case 5 facts: male, age 77 years, BP 170/95}

Retired, married, physically active man. No family history of cardiovascular disease. Non-smoker; drinks alcohol very modestly. Exercises regularly. Healthy diet. No other diseases. Presently has occasional discomfort in his left arm; in connection with this, his BP is measured and found to be high. Exercise stress ECG: normal. Blood pressure, as measured by a nurse, is $170 / 95$ and $150 / 85$; at the next visit, his BP is $155 / 80$. BMI: $22 \mathrm{~kg} / \mathrm{m}^{2}$. ECG: normal. Routine tests: NAD. Serum cholesterol: $6.2 \mathrm{mmol} / \mathrm{l}$.

\section{Case 5 risk assessment}

This is a case of medium cardiovascular risk with a medium hypertension levels. SCORE cannot be assessed in patients of this age. The ESC score is 2 .

\section{Case 5 results}

$58 \%$ of GPs wanted to start treatment with the aim of achieving the target BP (mean 140/86). Of these GPs, $58 \%$ selected thiazides as their first choice of treatment; $21 \%$ chose ACE inhibitors and 17\% beta blockers $17 \%$. $18 \%$ of GPs wanted to treat the raised cholesterol levels.

Summary of results Tendency to under-treat was particularly evident for cases 4 and 5, with just 70 and 58\% of GPs willing to treat these patients, respectively. Conversely, the present guidelines give no support for treatment in case no 1 with mild hypertension whereas the clinicians overwhelmingly chose to treat.

ACE inhibitors and thiazides were the most popular drug choices, in accordance with guidelines and local recommendations. For cases 1 and 5 (a young woman and an old man, respectively), thiazides were the preferred drugs. For case 2, who had a target organ damage (LVH), a rather low proportion of GPs (43\%) were choosing ACE inhibitors, which have best evidence for effectiveness for this condition.

There were a number of suggestions for treating other risk factors, with concentration on smoking cessation and non-pharmacological treatment of obesity. In case 3, a patient with type 2 diabetes, the responses show that the guidelines concerning treatment of hypertension when diabetes is present were well understood by GPs.

\section{Discussion}

The results of the present study, showed under- as well as overtreatment in the majority of the presented cases, emphasizing the need for better use of guidelines for the treatment of hypertension.

Case-based questionnaires aim to illustrate how judgment concerning treatment is made in cases of different severity. The cases provide a description of a real clinical challenge in a realistic clinical setting. It is important to receive a high response rate, and in order to achieve this the amount of information for the case presentation has to be reasonable. In this study, the participating GPs were randomly selected. Together with the high participation rate, this adds to the credibility of the results.

In addition, the cases were reasonably varied in terms of overall cardiovascular risk factor burden.

Our study has some limitations. A questionnaire is not the same as clinical practice, where decisions on treatment take place in concordance with the wishes of the patient, and in itself, the questionnaire may induce a more active approach than real life. Also, although guidelines do exist, there are no correct answers as such for individual cases. It should also be pointed out that the responders completed the questionnaires during office hours with a normal patient load and may thus not have had adequate time to consider the clinical implications for each case. This may have caused inconsistencies regarding the answers.

Perceived lack of time may also show the difficulty of implementing the SCORE system as a method for use in clinical practice. It could be used as a guideline, but not for automatically translating data from population studies to clinical practice. On the other hand, cases may be used as an educational method to increase the awareness of treatment options, as well as a method of priority [7].

Assessment by the ESH and SCORE methods has been questioned, particularly their failure to indicate the same CV risk levels [8]. For example, the SCORE system does not include diabetes as a risk factor. Also, further risk assessment tools tailored to primary care have recently been proposed [9]. Regarding the generalisability of the results, the answers came from a particular setting, but the cases used should be familiar in the Western world. The results show that correctly assessing risk is difficult, affecting the consequent choice of treatment.

Finally, our questionnaire has not been validated, which makes the reproducibility of the responses uncertain. It would be difficult to find a method of validation since all information of the patient cannot be provided.

Recently, the awareness-to-adherence model has been presented [10] in an attempt to explain failures to reach the blood pressure target. This model could also show that guidelines are on their own insufficient, and should be complemented by educational efforts.

Self-report studies have described treatment in clinical practice $[11,12]$. However, these studies have been limited to BP targets and the reasons for not intensifying 
treatment. Further questions, such as drug choices and familiarity with research methods, were addressed in a large survey [13]. Some geographic differences in attitudes concerning age and the cost of medication have also been reported [14]. When co-morbidity is taken into consideration, younger physicians are more likely to follow guidelines [15], a finding we could not confirm. This may also be true for non-pharmacological recommendations [16].

Although frequently commented by our responders, we have not quantified this particularly.

A case-based method could be of help in the assessment of judgments made by the clinicians. Indeed, this has been used for other diagnoses [17], but the use of the method is rare for the treatment of high BP, and thus we find our study adding to present knowledge.

The difficulty in showing adherence to guidelines due to different risk levels was described by Milchak et al [18]. Previous studies [19] have also shown limited correlation between increased number of risk factors and increased levels of treatment. Another study [20] showed that physicians tend to focus rather on diastolic blood pressure when choosing treatment, leaving systolic hypertension less well controlled. Our findings rather contradict this and thus, our findings confirm that there is much room for improvement in the assessment of CVD risk. E.g., this is illustrated in case 5 , where the low treatment rate may be due to reluctance to treat older patients, although this may be the group which would have the greatest benefit from treatment [21]. However, when it comes to treatment of the oldest, a Swedish study showed that lower systolic blood pressure may be associated with greater mortality in patients aged 85 or more. [22]

Factors causing reluctance to treat high BP may thus include hesitance about the guidelines, partly due to doubts concerning the effectiveness of additive treatment (referred to by some as "over-treatment"), difficulty in being up-to date, but also lack of time with patients and GPs concerns about lack of compliance.

The importance for adequate prevention for patients at risk is evident. This leads to the objective to increase adherence to guidelines, to put into practice what is considered an improved treatment, and in this process the cases in the questionnaire may be complemented by a structured study program showing the relative impact of different risk factors. Then it is of major importance that the guidelines give similar recommendations for treatment.

\section{Conclusions}

The present study confirmed the lack of adherence to present hypertension guidelines. Indeed, where additional risk factors are present, the guidelines do not seem to increase the tendency to initiate treatment. It may be easier to recognise an isolated $\mathrm{BP}$ value than to deal with more complex patient cases in which different risk factors should be taken into consideration.

For the future, since hypertension is common in primary care, we believe research emphasis should be put on efforts to increase the awareness and thus the adherence to guidelines in this setting. Computer-based tools allowing new - medical and educational- data to be readily available could prove useful for this purpose.

\section{Consent}

The study was approved by the Ethics committee of Lund University.

Competing interest

There are no financial or other conflicts of interest.

\section{Acknowledgements}

Financial support: The R\&D Department of Primary Care, Region Skåne and the Faculty of Medicine, Lund University.

\section{Author details}

${ }^{1}$ General Practice/Family Medicine, Department of Clinical Sciences, Faculty of Medicine, Lund University, Malmö, Sweden. ${ }^{2}$ The R\&D Department of Primary Care, Malmö, Region Skåne, Sweden. ${ }^{3}$ Cardiovascular Epidemiology, Department of Clinical Sciences, Faculty of Medicine, Lund University, Malmö, Sweden. ${ }^{4}$ Dalby Primary Health Care Centre, Skolgatan 1, S-240 10 Dalby, Sweden.

\section{Authors' contributions}

All authors have been active within the framework for cardiovascular prevention in Region Skåne. RE has been the main manuscript writer with co-work and input from PM. SG, KP and CN have participated in the discussions over cardiovascular prevention, the individual cases and have provided comments on the manuscript. $L$ have been the provider of the cases and evaluated them in reference to guidelines and has also participated actively in producing the text in the manuscript. All authors read and approved the final manuscript.

Received: 16 May 2011 Accepted: 26 April 2012

Published: 26 April 2012

\section{References}

1. 2003 European society of Hypertension-European Society of Cardiology guidelines for the management of arterial hypertension. J Hypertens 2003, 6:1011-1053.

2. Conroy RM, Pyörälä K, Fitzgerald AP, Sans S, Menotti A, De Backer G, De Bacquer D, Ducimetière $P$, Jousilahti $P$, Keil U, Niølstad I, Oganov RG, Thomsen T, Tunstall-Pedoe H, Tverdal A, Wedel H, Whincup P, Wilhelmsen L, Graham IM: SCORE project group. Estimation of ten-year risk of fatal cardiovascular disease in Europe: the SCORE project. Eur Heart J 2003, 24:987-1003.

3. Yusuf S, Hawken S, Ounpuu S, Dans T, Avezum A, Lanas F, McQueen M, Budaj A, Pais P, Varigos J, Lisheng L: INTERHEART Study Investigators. Effect of potentially modifiable risk factors associated with myocardial infarction in 52 countries (the INTERHEART study): case-control study. Lancet 2004, 364:937-952.

4. Moderately elevated blood pressure 2006. The Swedish Council on Technology Assessment in Health Care: Stockholm, 2006.

5. Hedblad B, Nerbrand C, Ekesbo R, Johansson L, Midlöv P, Brunkstedt I, Gyllerup S, Sträng B, Persson R, Janzon L: High blood pressure despite treatment: a cross-sectional primary healthcare-based study in southern Sweden. Scand J Prim Health Care 2006, 24:224-230.

6. Midlöv P, Ekesbo R, Johansson L, Gerward S, Persson KO, Nerbrand C, Hedblad $B$ : Barriers to adherence to hypertension guidelines among GPs in southern Sweden: a survey. Scand J Prim Health Care 2008, 26(3):154-159.

7. Herbert CP, Wright JM, Maclure M, Wakefield J, Dormuth C, Brett-MacLean P, Legare J, Premi J: Better Prescribing Project: a randomised controlled trial of the impact of case-based educational modules and personal 
prescribing feedback on prescribing for hypertension in primary care. Fam Pract 2004, 21:575-581.

8. Lengelé JP, Vinck WJ, De Plaen JF, Persu A: Cardiovascular risk assessment in hypertensive patients: major discrepancy according to ESH and SCORE strategies. J Hypertens 2007, 25:757-762.

9. D'Agostino RB Sr, Vasan RS, Pencina MJ, Wolf PA, Cobain M, Massaro JM, Kannel WB: General cardiovascular risk profile for use in primary care: the Framingham Heart Study. Circulation 2008, 117:743-753.

10. Heneghan C, Perera R, Mant D, Glasziou P: Hypertension guideline recommendations in general practice: awareness, agreement, adoption, and adherence. Br J Fam Pract 2007, 57:948-952.

11. Frijling BD, Spies TH, Lobo CM, Hulscher ME, an Drenth BB, Braspenning JC, Prins SA, van der Wouden JC, Grol RP: Blood pressure control in treated hypertensive patients: clinical performance of general practitioners. $\mathrm{Br} J$ Gen Pract 2001, 512:9-14

12. Ferrari $P$, Hess $L$, Pechere-Bertschi A, Muggli F, Burnier M: Reasons for not intensifying anti-hypertensive treatment (RIAT): a primary care anti-hypertensive intervention study. J Hypertens 2004, 22:1221-1229.

13. Hyman DJ, Pavlik VN: Self-reported hypertension treatment practices among primary care physicians: blood pressure thresholds, drug choices, and the role of guidelines and evidence-based medicine. Arch Intern Med 2000, 160:2281-2286.

14. Troein M, Arneson T, Råstam L, Pirie PL, Selander S, Luepker RV: Reported treatment of hypertension by family physicians in Sweden and Minnesota: a physician survey of practice habits. J Intern Med 1995, 238:215-221.

15. Mehta SS, Wilcox CS, Schulman KA: Treatment of hypertension in patients with comorbidities: results from the study of hypertensive prescribing practices (SHyPP). Am J Hypertens 1999, 12:333-340.

16. Arroll B, Jenkins S, North D: Non-pharmacological management of hypertension: results from interviews with 100 general practitioners. Hypertens 1996, 14:773-777.

17. Beaulieu MD, Brophy J, Jacques A, Blais R, Battista RN, Lebeau R: Physicians' attitudes to the pharmacological treatment of patients with stable angina pectoris. QJM 2005, 98:41-51.

18. Milchak J, Carter BL, Ardery G: Measuring adherence to practice guidelines for the management of hypertension: an evaluation of the literature. Hypertension 2004, 44:602-608.

19. Kannel WB, D'Agostino RB, Sullivan L, Wilson PW: Concept and usefulness of cardiovascular risk profiles. Am Heart J 2004, 148:16-26.

20. Fagard RH, van den Emden M: Treatment and blood pressure levels in isolated hypertension and diastolic hypertension in primary care. I Hum Hypertens 2003, 17:680-687.

21. Beckett NS, Peters R, Fletcher AE, Staessen JA, Liu L, Dumitrascu D, Stoyanovsky V: Treatment of hypertension in patients 80 years of age or older. N Engl J Med 2008, 358:1887-1898.

22. Molander L, Lövheim H, Norman T, Nordström P, Gustafson Y: Lower Systolic Blood Pressure is associated with greater mortality in people aged 85 and older. J Am Geriatr Soc 2008, 56:1853-1859.

doi:10.1186/1471-2296-13-34

Cite this article as: Ekesbo et al:: Lack of adherence to hypertension treatment guidelines among GPs in southern Sweden-A case report-based survey. BMC Family Practice 2012 13:34.

\section{Submit your next manuscript to BioMed Central and take full advantage of:}

- Convenient online submission

- Thorough peer review

- No space constraints or color figure charges

- Immediate publication on acceptance

- Inclusion in PubMed, CAS, Scopus and Google Scholar

- Research which is freely available for redistribution

Submit your manuscript at www.biomedcentral.com/submit 